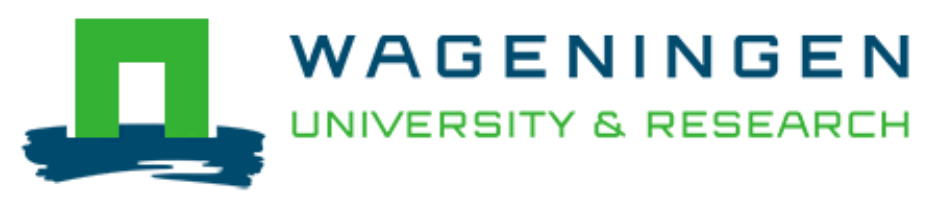

Coarse-Grained Dendrimers in a Good Solvent: Comparison of Monte Carlo Simulations, Self-Consistent Field Theory, and a Hybrid Modeling Strategy

Bergsma, J., van der Gucht, J., \& Leermakers, F. A. M.

This article is made publically available in the institutional repository of Wageningen University and Research, under article 25fa of the Dutch Copyright Act, also known as the Amendment Taverne.

Article $25 \mathrm{fa}$ states that the author of a short scientific work funded either wholly or partially by Dutch public funds is entitled to make that work publicly available for no consideration following a reasonable period of time after the work was first published, provided that clear reference is made to the source of the first publication of the work.

For questions regarding the public availability of this article, please contact openscience.library@wur.nl.

Please cite this publication as follows:

Bergsma, J., van der Gucht, J., \& Leermakers, F. A. M. (2019). Coarse-Grained Dendrimers in a Good Solvent: Comparison of Monte Carlo Simulations, SelfConsistent Field Theory, and a Hybrid Modeling Strategy. Macromolecular Theory and Simulations, [1800064]. https://doi.org/10.1002/mats.201800064 


\title{
Coarse-Grained Dendrimers in a Good Solvent: Comparison of Monte Carlo Simulations, Self-Consistent Field Theory, and a Hybrid Modeling Strategy
}

\author{
Johan Bergsma, * Jasper van der Gucht, and Frans A. M. Leermakers*
}

Recently, a hybrid method has been developed, wherein the positions of some polymer segments are constrained to a small volume. The volume's position is sampled with a Monte Carlo algorithm. The distribution of the remaining segments is determined with Scheutjens-Fleer self-consistent field theory (SF-SCF). This incorporates thermal fluctuations into the model. Here it is investigated whether this also leads to an improved treatment of the excluded volume interactions. Dendrimers, with $f=1 \cdots 7$ generations, $g=2 \cdots 5$ spacers per branch point, and spacers of 20 or 50 segments, are used as a model system. The focus is on the radius of gyration, the asphericity, the radial density, and the end point and first branch point distribution. As expected, both SCF methods underestimate the radius of gyration due to underestimating the short range excluded volume. The SF-SCF model, however, also gives a slightly different scaling and, in contrast to the other models, also predicts a bimodal distribution for first generation branch points for large $f$ and $g$. This difference is attributed to overestimating the excluded volume at long ranges. As the hybrid method does not show this, localizing just a few segments largely compensates this shortcoming in the SF-SCF theory.

\section{Introduction}

Inhomogeneous polymeric materials are of significant technological interest. These materials range from simple polymeric solutions to polymeric micelles and (double) gel networks. They can be used as absorbents, drug carriers in nanomedicine, ${ }^{[1]}$ or to modify rheological behavior, to name just a few of their applications. To be able to design the polymers with which these materials can be created, it is important to understand how the properties of the polymers affect the behavior of these materials. Mean field theory, which evaluates the conformational degrees of freedom using a diffusion-like equation, is a popular way to study these systems as it is computationally very efficient. ${ }^{[2-9]}$

Dr. J. Bergsma, Prof. J. van der Gucht, Prof. F. A. M. Leermakers

Physical Chemistry and Soft Matter

Wageningen University \& Research

Stippeneng 4, 6708 WE, Wageningen, The Netherlands

E-mail: Johan.Bergsma@gmx.com; Frans.Leermakers@wur.nl

The ORCID identification number(s) for the author(s) of this article can be found under https://doi.org/10.1002/mats.201800064.

DOI: $10.1002 /$ mats.201800064
The accuracy of the theory is good for melts where excluded volume correlations are screened, ${ }^{[10]}$ but not so good in dilute cases where these correlations are important and we therefore do not know for sure which results of the self-consistent field (SCF) theory can be trusted. More detailed methods such as Molecular dynamics and Monte Carlo simulations do take these correlations into account but sample only one configuration at a time and therefore take more computation time. There are only a few strategies in between these limits of which the single chain mean field method is a notable example. ${ }^{[6,11]}$ With this method the intra molecular interactions are taken into account explicitly while the interactions between separate molecules are calculated in a mean field manner.

Recently, we have introduced a computational strategy to study spatially inhomogeneous polymeric systems wherein we combine the mean field Scheutjens Fleer self-consistent field theory (SF-SCF) with a Monte Carlo (MC) algorithm. ${ }^{[12,13]}$ This method differs from a previous combination of a Monte Carlo model with the SF-SCF theory by Charlaganov et al. ${ }^{[14]}$ They first calculated an approximate free energy based on estimated interaction potentials between the sites that are moved with a Monte Carlo algorithm. Based on this free energy the Monte Carlo move was accepted or rejected. If the move was accepted they calculated a more accurate free energy with the SF-SCF method and used this free energy to weigh the state. If the difference in free energy between these free energies is small $\left(<1 k_{B} T\right)$ this method is quite effective. If the difference is, however, bigger, the method presented in this article in which we use the free energy obtained from the SF-SCF theory for the acceptance criterion will be more efficient.

We used this recently introduced method to simulate a physical gel of telechelic polymers. The polymer distributions were computed with the SF-SCF method, with the constraint that the polymer ends lie within so-called nodes (which are micellarlike cores that have specified locations). In turn, the nodes were moved with a Monte Carlo algorithm, which uses the SCF free energy of the system in the "acceptance" step. Compared to classical SCF, calculations of this type are computationally expensive, as for each set of node positions the distribution of the telechelic polymers has to be recalculated. The computation 
time of the SCF-part of the simulation scales with the volume of the system, but not with the number of chains in it. This possibly counterintuitive feature makes the model relatively efficient for systems with a high polymer density such as the physical gels of telechelic polymers we studied previously.

The corresponding computations for a chemically crosslinked gel, however, require the strict coupling of chain ends to specified nodes (cross-links) and this makes the hybrid SCF method computationally even more challenging. In this case, the computation time scales with the number of chains (or chain fragments) in the system. We show that, by using subboxes and implementing the computations on a GPU, it is still feasible to develop and execute a hybrid method, with explicit chemical cross-links, that combines the Monte Carlo and selfconsistent field methods (MC-SCF). Here, we implement this method not for a chemically cross-linked gel, but for dendrimer molecules, which may be seen as micro-gels.

One of the main reasons for developing hybrid MC-SCF methods is to investigate the shortcomings of the computationally extremely inexpensive SF-SCF method. With SF-SCF theory one uses a one-gradient spherical coordinate system (cell model) and the averaged properties of a system are predicted. In reality, deviations from spherical symmetry exist, especially for molecules with few internal branches, most notably linear chains. The SF-SCF model cannot describe the shape of individual macromolecule conformations and properties that depend on these.

Other limitations of SCF exist. One issue is that every segment in SCF "feels" an averaged local density instead of the real segment positions. A segment therefore does not feel that locally the density is higher due to segments that are close to it in the molecule, and whose positions are correlated. ${ }^{[15]}$ This allows conformations that fold back onto themselves. As a result, the local excluded volume and thus the local stretching is underestimated.

At the same time the long range steric repulsion is overestimated within SCF, as it is assumed that all the segments are distributed homogeneously within each lattice layer. In reality, the excluded volumes of segments that are close to each other in the molecule will overlap and the total excluded volume of the chain is thus smaller. ${ }^{[15]}$ The number of possible chain conformations is therefore higher as fewer chain conformations overlap.

In the newly formulated hybrid method, some of the segments are localized on a specific lattice site and moved with a Monte Carlo algorithm. The other segments are still treated with the SF-SCF machinery. This way the correlation in position between segments close to each other in the molecule is increased. This should cause segments close to each other in the molecule to feel their excluded volume better, and at the same time reduce the excluded volume effect between segments far away from each other in the molecule. This should alleviate the problems of the SF-SCF cell model, while still keeping the number of degrees of freedom that need to be sampled with the Monte Carlo algorithm limited.

In contrast to our previous articles, ${ }^{[12,13]}$ we now need to specify at which point a chain-fragment starts and ends, as the spacers in a dendrimer are attached to each other with chemical bonds. As a result, each spacer needs its own propa- gator. Because this propagator can only have non zero values in a small region near the branch points, we introduce small sub-boxes, so we only need to calculate the propagator in areas where it can have a non-negligible value. In the current implementation on a GPU, it is convenient to make all sub-boxes the same size and shape. The size of the sub-boxes is chosen in such a way that for all combinations of start and end points, the probability of the chain going out of the box is negligible. The efficiency can probably be improved by modifying the size of the box as a function of the positions of the start and end points and the propagator step.

We used dendrimers as a model system, to determine how effective this new model is with respect to accounting for excluded volume correlations. The reason for choosing dendrimers is that excluded volume interactions play an important role in them and there is already some literature to compare our findings with. ${ }^{[4,16-19]}$ We compared three different methods: i) The cell model, that employs the classical SF-SCF equations ii) the newly developed MC-SCF model, and iii) as a reference, a Monte Carlo model of a hard sphere freely jointed chain (MC-FJC). We modeled systems with $f=2$ up to $f=5$ branches per node and up to $g=7$ generations. Results for a large number of generations and high degree of branching are rare in the literature. We include these because the MC-SCF method and especially the classical SF-SCF results are expected to improve for these molecules. Due to the many internal branches, the molecules can not deviate much from the overall spherical geometry. The cell model, which assumes spherical symmetry, should thus be able to describe such a molecule well.

\section{Experimental Section}

\subsection{Dendrimer Definition}

Dendrimers have a hierarchical structure and an illustration of such a molecule is presented in Figure 1. They "start" with a central (root) segment. To this central segment, $f$ spacers are connected each having $S$ segments. At the end of all these spacers there is a next segment that functions as a branch point. From this and each subsequent branch point there are $f-1$ new spacers of length $S$. The number of generations $g$ is defined as the number of branch points that are encountered starting from the center and taking any path to the free ends of the molecule. In this process, the central segment is also counted. At the end of the spacers of the final generation there is an extra terminal segment. This means that the length of the path from the center to any of the free ends is $N=g(S+1)+1$ segments long. The number of end points is given by $n_{\mathrm{e}}=f(f-1)^{\mathrm{g}-1}$. The number of spacers (arms) (including the outer ones) in the dendrimer equal $n_{\text {spacer }}=\sum_{j=1}^{g} f(f-1)^{j-1}$ and the number of branch points is $n_{\text {branch }}=1+\sum_{j=1}^{g-1} f(f-1)^{j-1}$. The total number of segments in the dendrimer is given by $N=n_{\text {branch }}+n_{\text {spacer }} S+n_{\mathrm{e}}$.

The functionality $f$ was varied from 2 to 5 and two values were used for the spacer length, $S=20$ and $S=50$. For $f>2$, the number of generations modeled with the MC-SCF method was limited by the 3 GB of memory available on the GPU. It 


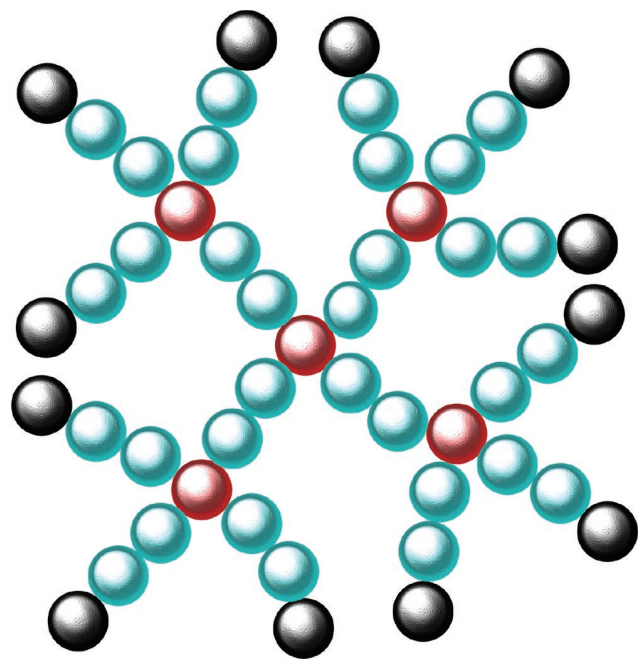

Figure 1. An example of a dendrimer with $f=4$ arms per branch point, $g=2$ generations, and spacer length (number of segments in between branch points) $S=2$. The spacers are cyan (lightest), the branch points are red, and the end points are black (darkest).

ranged from $g=7$ for $f=3$ and $n=20$ to $g=2$ for $f=5$ with $S=$ 50. This limitation could be lifted by implementing memory efficient algorithms. As this would have increased the computational cost even more, the parameter space was restricted to the limiting values that could be treated by MC-SCF.

\subsection{Freely Jointed Chain Model with Excluded Volume (MC-FIC)}

As a reference model, a dendrimer composed of hard sphere segments, each with diameter $d$, connected by bonds of length $l$ that could freely rotate, was used. In the simulations, the bond length was taken as the unit for distance, that is, $l=1$ by definition. For ease of comparison with the SCF models, the diameter of the beads was chosen such that the maximum packing density of the beads was the same as in the SCF model. The diameter of the beads was therefore chosen as:

$d=\left(\frac{1}{4 \sqrt{2}}\right)^{\frac{1}{3}}=0.561$

In hindsight the beads overlapped a bit when $l=1$. The real maximum packing fraction was therefore slightly higher. This was not a real issue because it was expected that a pre-factor was needed to match the outcomes of the SCF models with those of the MC-FJC model anyway.

Similar to Chen et. al., ${ }^{[18]}$ a Monte Carlo protocol with pivot moves was used to generate new dendrimer configurations. In a pivot move, a segment of the dendrimer was randomly selected. This segment and the outward part of the dendrimer attached to this segment were rotated around the segment one step closer to the center along the contour of the dendrimer. The axis around which the rotation took place was chosen randomly from the three perpendicular axes. The angle of rotation was randomly chosen from the range of 0 to $2 \pi$. If there was no overlap between the segments, after the rotation, the move was accepted. When the move was rejected, the original conformation was counted again in the statistical averaging.

For dendrimers with a large number of segments, the probability that pivot moves of segments close to the center were accepted became very small. For large dendrimers, an extra move therefore was introduced for the segments between the center of the dendrimer and the first branch points. This move consisted of rotating the segment by a random angle around the axis through the two segments to which it was attached. Whether this move or a pivot move was done for these segments was determined randomly with each having a 50\% chance. The probability that these segments were chosen was also doubled.

Due to the accumulation of rounding errors, the bond lengths would over time start to deviate from unity. At regular intervals, the bond lengths were therefore readjusted to unity. Occasionally, this caused two segments to overlap. In that case, the particle closest to the center along the contour was moved away from the other particle until the distance between them was 1.00001. After this, the bond lengths were again readjusted to length unity. The whole process was repeated till all bonds had length one and there was no overlap.

The procedure was tested with an ideal dendrimer (without excluded volume) and the correct average end point distance and bond angle distribution were found indicating that the method was implemented correctly and that all configurations were visited with the correct a priori probability.

The simulations were started by generating a dendrimer with segments with zero radius. Starting from the previous segment, a number of new positions were randomly generated at distance $l$. Subsequently, the position that was most distant from the $s-1$ beads that were already in place was chosen. An "inverse distance" $D\left(\mathbf{r}_{s}\right)$ was defined as

$$
D\left(\mathbf{r}_{s}\right)=\sum_{i=1}^{s-1} \frac{1}{\left\|\mathbf{r}_{i}-\mathbf{r}_{s}\right\|}
$$

and the position with the smallest $D\left(\mathbf{r}_{s}\right)$ value was chosen. Here $\mathbf{r}_{s}$ was the position of the bead $s$.

Once the dendrimer structure was generated, the Monte Carlo machinery was started. In addition to the standard Monte Carlo moves, the radius was increased to 0.561 if this would not result in overlap with other beads. Once all the beads had the correct volume, an equilibration run was done with a tenth of the number of steps used for data collection. The number of Monte Carlo steps taken varied between the different simulation runs. An overview of all the simulation runs and the number of Monte Carlo steps taken, together with the calculated values for the radius of gyration, the end point distance, and the asphericity, is given in Supporting Information.

\subsection{SF-SCF Theory}

The classical SF-SCF theory ${ }^{[2,12,20-28]}$ has been described in the literature in detail. Here, the focus was only on the main issues and ideas that were relevant for the modeling of dendrimers in the MC-SCF and cell model implementations. 
Just as in the freely jointed chain model, macromolecules are described as a string of segments connected by bonds of fixed length, equal to the segment diameter. The chains "live" in a discretized world, also known as a lattice. The size of the lattice sites is equal to the bond length, the segments thus fit exactly in the lattice sites. Segments that are connected in the molecule will be in neighbouring lattice sites. The longer ranged correlations between segment positions are ignored; this is also known as the Markov chain approximation. In contrast to the MC-FJC model, chain backfolding is thus allowed.

In this lattice, a mean field approximation was applied. This meant that the average volume fractions in a lattice site $\varphi(\mathbf{r})$ were determined based on the interactions with the average environment. Next to the volume fractions $\varphi(\mathbf{r})$, each lattice site also had a segment potential $u(\mathbf{r})$ for each segment type. The segment potential at coordinate $\mathbf{r}$ represented the energy needed to bring a segment from the bulk to coordinate $\mathbf{r}$, when this segment was not (yet) connected to other segments in a chain. For convenience, the potentials were expressed in units $k_{B} T$.

The segment potentials implemented the interactions between the segments. The segment-interactions were accounted for with the Bragg-Williams mean field approximation and described by the Flory-Huggins $\chi$ interaction parameter. As the focus was on good solvent conditions, $\chi=0$. In this case the only remaining contribution to the segment potential came from the incompressibility condition. This means that the segment potentials were chosen such that the sum of the volume fractions of the solvent, $\varphi_{O}(\mathbf{r})$ and the dendrimer $\varphi_{D}(\mathbf{r})$ added up to unity, $\varphi_{D}(\mathbf{r})+\varphi_{O}(\mathbf{r})=1$ at each coordinate $\mathbf{r}$. To determine the values for the segment potentials, the volume fractions are required. As the volume fractions in turn depend on the segment potentials, a chicken and egg problem is encountered. To circumvent this, the segment potentials were initially guessed, usually $u(\mathbf{r})=0$. With this guess, the volume fractions were computed. This began with the evaluation of socalled segment statistical weights $G(\mathbf{r})$, which are given by:

$G(\mathbf{r})=e^{-u(\mathbf{r})}$

The segment statistical weight is the contribution a segment at the specified coordinate would make to the statistical weight of polymer conformations that pass through the lattice site. The solvent consisted of freely dispersed monomers and therefore their volume fractions were proportional to the segment statistical weights and were determined with:

$\varphi_{O}(\mathbf{r})=G(\mathbf{r})$

This is equivalent to $\varphi_{O}(\mathbf{r})=\frac{N_{O}}{q_{O}} G(\mathbf{r})$. The latter equation is numerically more stable. Here $N_{O}$ is the number of solvent molecules in the system and $q_{0}=\sum_{\mathbf{r}} G(\mathbf{r})$.

Determining the polymer segment distribution is more complicated. For this, statistical weights of sets of conformations of parts of the polymer have to be combined. The so-called end point distribution functions $G\left(\mathbf{r}_{\mathbf{t}}, t \mid \mathbf{r}_{\mathbf{b}}, b\right)$ are used to do this. These contain the combined statistical weight of all possible and allowed conformations of a chain fragment beginning with segment number $b$ at coordinate $\mathbf{r}_{b}$ and terminating with segment $t$ at coordinate $\mathbf{r}_{\mathbf{t}}$. These end point distributions are generated using a propagator formalism which employs segment statistical weights as a key ingredient. The segment density, $\varphi(\mathbf{r}, s)$, for segment $s$ at coordinate $\mathbf{r}$ is found by combining all end point distributions that could reach segment s. The details of this formalism depend on the molecular topology of the chains and on the constraints that were imposed. This information differed between the MC-SCF hybrid and the cell model. Therefore the details are given in the following separate subsections.

Once the volume fractions are determined, the segment potentials in turn can be calculated. If the sum of the volume fractions is larger than unity, the densities need to be suppressed. This is implemented by increasing the potentials. The inverse should happen when the volume fractions are below unity. Hence, the segment potential at each iteration $k+1$ could be updated from values found at iteration $k$ :

$u^{k+1}(\mathbf{r})=u^{k}(\mathbf{r})+\eta\left(1-\frac{1}{\varphi_{0}^{k}(\mathbf{r})+\varphi_{D}^{k}(\mathbf{r})}\right)$

where $\eta$ lies in the range $0<\eta<1$ and is chosen such that convergence is achieved. These equations can be iterated over until the sum of the volume fractions in each lattice site has converged to one.

In practice, a target function that combines the incompressibility relation with a self-consistency of the potentials ${ }^{[23]}$ and more sophisticated algorithms which converge faster than a simple steepest descent algorithm are used. An example is the DIIS algorithm ${ }^{[29]}$ which converges routinely and efficiently in 10 to 100 iteration steps to the SCF solution to a precision of 7 significant digits.

\subsection{MC-SCF}

In the MC-SCF model, a 3D simple cubic lattice is used where the coordinates are given by $\mathbf{r}$. The total number of lattice sites in each simulation is given by $m^{3}$, where $m$ is the total number of sites in each of the three directions $x, \gamma, z$. The idea is that the dendrimer are split up in spacers, which lie in between the branch points and between branch and end points. For each dendrimer, there are $n_{\text {spacer }}$ spacers for which at each point during the simulation the start and end segments have to lie next to the appropriate branch and end points. The initial positions of these branch points were generated using a random walk on the lattice, which could be biased to move away from its starting position. Starting from the root segment, $S+1$ steps were done in a random direction to reach the position of the first generation of branch points. This process was repeated until finally the positions of the end points were determined. Obviously, it was checked that the branch and end points were not placed on top of each other. In a few cases, this did not work and instead the positions of the branch and end points were remapped from the MC-FJC model onto the lattice.

For each spacer $i$, a separate propagator is needed to generate the densities of this fragment $\varphi_{i}(\mathbf{r})$ from the segment potentials $u(\mathbf{r})$. There exists an excellent opportunity to do this in parallel 
because the calculation of the propagator of one spacer is independent of those of other spacers. It is also clear that the propagator does not need to be developed in the entire volume, as spacers could only contribute to the density in the vicinity of the branch and end points they are connected to. Therefore, for each spacer $i$, a sub-volume $v_{i}=M^{3}$, called sub-box, was specified with sub-coordinates $R_{i}=\left(X_{i}, Y_{i}, Z_{i}\right)$ each running from $X_{i}, Y_{i}, Z_{i}=1, \ldots, M$. The position of this sub-box was chosen such that the center of this sub-volume lay exactly between the branch/end points.

It is clear that the sub-box $M$ could be much smaller than the main box $m$. For $S=20$, the sub-box size was $M=20$; for $S=50$, a sub-box size of $M=37$ was used. The latter was smaller than the maximum extension of the polymer. The required entropyloss to stretch the polymer to this size is, however, very high and stretching beyond 25 lattice sites was not observed. The small boxes had reflecting boundary conditions even though these conditions would not significantly influence the results because the sub-box size $M$ was so large that conformations rarely "hit" the sub-box boundaries. For each sub-box $i$, the subbox coordinates were filled with the segment potentials from the main volume: $u_{i}\left(\mathbf{R}_{\mathbf{i}}\right)=u\left(\mathbf{r}_{i}+\mathbf{R}_{i}\right) \forall \mathbf{R}_{i}$. Here $\mathbf{r}_{i}$ is the position of the origin of the sub-box $i$ on the main lattice.

Also the position $\mathbf{r}_{0}^{\mathrm{i}}$ of the first segment $s=0$ and the position $\mathbf{r}_{\mathbf{s}+1}^{\mathrm{i}}$ of the last segment $s=S+1$ were transferred to the appropriate sub-box. When a sub-box crossed the periodic boundaries in the main system, the periodic image was used to transfer the information to the sub-box. The full set of branch and end points is indicated by $\left\{\mathbf{r}_{0}, \mathbf{r}_{\mathrm{S}+1}\right\}$.

The first step was to compute the segment statistical weights $G(\mathbf{r})$. All branch and end points, however, already occupied their lattice site and hence no other segment could go there. Therefore it was imposed that the segment statistical weight was zero at these sites:

$G(\mathbf{r})= \begin{cases}e^{-u(\mathrm{r})}, & \mathbf{r} \notin\left\{\mathbf{r}_{0}, \mathbf{r}_{\mathrm{s}+1}\right\} \\ 0 & \mathbf{r} \in\left\{\mathbf{r}_{0}, \mathbf{r}_{\mathrm{S}+1}\right\}\end{cases}$

With these segment statistical weights, the end point distribution functions could becalculated. Two complementary end point distributions were found recursively, one by a so-called forward and the other by a backward propagator. The forward procedure was initialized by setting $G_{i}\left(\mathbf{r}, 0 \mid \mathbf{r}_{0}^{\mathrm{i}}, 0\right)=1$ when $\mathbf{r}=\mathbf{r}_{0}^{\mathrm{i}}$ for spacer $i$ and 0 otherwise.

$G_{i}\left(\mathbf{r}, s \mid \mathbf{r}_{0}^{\mathrm{i}}, 0\right)=G(\mathbf{r})\left\langle G_{i}\left(\mathbf{r}, s-1 \mid \mathbf{r}_{0}^{\mathrm{i}}, 0\right)\right\rangle$

In Equation (7), the angular brackets implement the averaged value over the neighbouring lattice sites. For a simple cubic lattice, this gives:

$$
\begin{aligned}
\langle G(\mathbf{r})\rangle & =\langle G(X, Y, Z)\rangle \\
& =\frac{1}{6}((G(X+1, Y, Z)+G(X-1, Y, Z)+G(X, Y+1, Z) \\
& +G(X, Y-1, Z)+G(X, Y, Z+1)+G(X, Y, Z-1))
\end{aligned}
$$

where it is understood that only the spatial coordinate was kept in the notation, that is, $G(\mathbf{r})$ is a shorthand for $G\left(\mathbf{r}, s \mid \mathbf{r}_{0}, 0\right)$. The "jump" to the last segment $s=S+1$ is given by
$G_{i}\left(\mathbf{r}, S+1 \mid \mathbf{r}_{0}^{\mathrm{i}}, 0\right)= \begin{cases}\left\langle G_{i}\left(\mathbf{r}, S \mid \mathbf{r}_{0}^{\mathrm{i}}, 0\right)\right\rangle, & \mathbf{r}=\mathbf{r}_{\mathbf{S}+1}^{\mathrm{i}} \\ 0 & \mathbf{r} \neq \mathbf{r}_{\mathbf{S}+1}^{\mathrm{i}}\end{cases}$

The backward propagator started from the other end of the spacer and hence was started with $G_{i}\left(\mathbf{r}, S+1 \mid \mathbf{r}_{\mathrm{S}+1}^{\mathrm{i}}, S+1\right)=1$ when $\mathbf{r}=\mathbf{r}_{\mathrm{S}+1}^{\mathrm{i}}$ and zero otherwise. The calculation of the backward propagator is continued with:

$G_{i}\left(\mathbf{r}, s \mid \mathbf{r}_{\mathbf{s}+1}^{\mathrm{i}}, S+1\right)=G(\mathbf{r})\left\langle G_{i}\left(\mathbf{r}, s+1 \mid \mathbf{r}_{\mathbf{s}+1}^{\mathrm{i}}, S+1\right)\right\rangle$

until at $s=0$

$G_{i}\left(\mathbf{r}, 0 \mid \mathbf{r}_{\mathrm{S}+1}^{\mathrm{i}}, S+1\right)= \begin{cases}\left\langle G_{i}\left(\mathbf{r}, 0 \mid \mathbf{r}_{\mathrm{S}+1}^{\mathrm{i}}, S+1\right)\right\rangle, & \mathbf{r}=\mathbf{r}_{0}^{\mathrm{i}} \\ 0 & \mathbf{r} \neq \mathbf{r}_{0}^{\mathrm{i}}\end{cases}$

The volume fraction $\varphi(\mathbf{r}, s)$ of segment $s$ in lattice site $r$ was proportional to the sum of the statistical weights of all the chain conformations that passed through lattice site $r$ with segment $s$. Each chain conformation going from segment 0 to segment $s$ could be combined with any of the chain conformations going from $S+1$ to $s$. The volume fraction distribution of segments $s=0, \ldots, S+1$ for the fragment $i$ was therefore given by the product of two end point distribution functions, one computed starting from segment $s=0$ and the other by starting with segment number $S+1$, both ending with segment $s$ at the same coordinate $\mathbf{r}$

$\varphi_{i}(\mathbf{r}, s)= \begin{cases}\frac{1}{q_{i}} \frac{G_{i}\left(\mathbf{r}, s \mid \mathbf{r}_{0}^{\mathrm{i}}, \mathbf{0}\right) G_{i}\left(\mathbf{r}, s \mid \mathbf{r}_{\mathbf{S}+1}^{\mathrm{i}}, \mathbf{S}+1\right)}{G(\mathbf{r})}, & \mathbf{r} \notin\left\{\mathbf{r}_{0}, \mathbf{r}_{\mathbf{S}+1}\right\} \\ 0 & \mathbf{r} \in\left\{\mathbf{r}_{0}, \mathbf{r}_{\mathbf{S}+1}\right\}\end{cases}$

where $\mathbf{r}_{0}^{\mathrm{i}}$ and $\mathbf{r}_{\mathbf{s + 1}}^{\mathrm{i}}$ are the locations of the two ends of spacer $i$. In Equation (12), the division by $G(\mathbf{r})$ is necessary because the weight for segment $s$ is accounted for in both end point distribution functions that are in the numerator. Obviously, there can not be any other segment in the specified branch points, therefore $\varphi_{i}(\mathbf{r}, s)=0$ when $\mathbf{r} \in\left\{\mathbf{r}_{0}, \mathbf{r}_{\mathbf{S}+1}\right\}$. The normalization by $\frac{1}{q_{i}}$ ensured that there was exactly one segment $s$ of molecule fragment $i$ in the sub-box. Here $q_{i}$ is the chain partition function, which is the combined statistical weight of all possible and allowed conformations of the chain fragment. It is given by:

$q_{i}=G\left(\mathbf{r}_{\mathrm{s}+1}^{\mathrm{i}}, S+1 \mid \mathbf{r}_{0}^{\mathrm{i}}, 0\right)$

The partition function for the solvent is:

$q_{O}=\sum_{\mathbf{r}} G(\mathbf{r})$

The overall volume fraction distribution of the dendrimer was found by adding up the segment distributions of all the spacers:

$\varphi(\mathbf{r})=\sum_{i} \sum_{s=1}^{S} \varphi_{i}(s, \mathbf{r})$ 
when $\mathbf{r} \notin\left\{\mathbf{r}_{0}, \mathbf{r}_{\mathrm{S}+1}\right\}$ and $\varphi(\mathbf{r})=1$ otherwise. Again, the segment potentials were adjusted iteratively until the volume fractions of the solvent and the dendrimer added up to unity at each coordinate in the main box. This SCF solution was obtained with at least five significant digits.

At this point, all the information needed to determine the free energy $F$ is available.

$F=-\ln q_{O}-\sum_{i} \ln q_{i}-\sum_{\mathbf{r} \notin\left\{\mathbf{r}_{0}, \mathbf{r}_{\mathbf{S}+1}\right\}} u(\mathbf{r})$

This free energy was then used as the classical "energy" in the MC formalism. This meant that the Metropolis acceptance was taken with the probability $p=e^{-\Delta F}$ when $\Delta F>0$ and $p=$ 1 otherwise. Here $\Delta F=F_{\text {new }}-F_{\text {old }}$. For the Monte Carlo trial step, a translational move was implemented. A known feature of the simple cubic lattice is that for a specified start and stop site, one either needs an odd or an even number of steps. This implied that if the starting coordinate was shifted by one site, the walks that connected these points went from having an even to an odd number of steps or vice versa. As all spacers had a fixed length $S$, the one-step trials were forbidden. Therefore, it was necessary to move any point an even number of steps. In the translational trial step, a branch point or an end point was selected randomly and its position was changed, by one lattice site in a random direction, twice.

The number of branch/end points that were moved within one trial step was optimized during the equilibration run, such that the acceptance probability times the number of branch/ end points moved was maximum. During data gathering the number of branch/end points that were moved was fixed. An equilibration run of 10000 Monte Carlo steps was done first, which was followed by data gathering for 90000 steps. Such simulations ran many days and therefore longer simulations were not feasible for the largest dendrimers. Optimization of the calculation efficiency might improve the speed significantly and in that case better averages could be obtained.

\subsection{Cell Model}

In the cell model, ${ }^{[30,31]}$ the system is assumed to be spherically symmetric. This is implemented by using a one-gradient spherical coordinate system. In such a coordinate system, lattice sites lie in concentric layers of thickness $l$ which are numbered $r=1, \ldots, m$. There is just one value of the segment potential and the volume fractions per layer. This greatly reduces the computational cost.

In the spherical geometry the number of lattice sites in each layer grows roughly quadratically: $L(r)=V(r)-V(r-1) \propto r^{2}$, with the dimensionless volume $V(r)=\frac{4}{3} \pi r^{3}$. The root segment of the dendrimer is pinned to $r=1$. The upper limit $r=m$ is larger than the longest path in the dendrimer $g(S+1)$, so the polymer density at the upper bound is zero. Just as for the simple cubic lattice, each lattice site is assumed to have $Z=6$ nearest neighbours. One in the layer above, one in the layer below and four in the same layer. Because the outer surface area is larger than the inner surface area, the a priori probability to go outward is larger than the probability to go inward. Therefore the following equations are used:

$$
\begin{aligned}
& \lambda_{+}(r)=\frac{4 \pi r^{2}}{6 L(r)} \\
& \lambda_{-}(r)=\frac{4 \pi(r-1)^{2}}{6 L(r)} \\
& \lambda_{0}(r)=1-\lambda_{+}(r)-\lambda_{-}(r)
\end{aligned}
$$

Here, $\lambda_{+}(r)$ is the a priori probability to go from $r$ to $r+1$, $\lambda_{-}(r)$ the probability to go from $r$ to $r-1$ and $\lambda_{0}(r)$ the probability to go to a lattice site in the same layer $r$.

These probabilities were used in the propagators. In the spherical coordinate system, the side fraction is given by:

$\langle G(r)\rangle=\lambda_{-}(r) G(r-1)+\lambda_{0}(r) G(r)+\lambda_{+}(r) G(r+1)$

wherein $G(r)$ is a shorthand for the appropriate end point distribution functions. Symmetric dendrimers have the property that all paths from the root segment to any of its ends are equally long. The segments of one such path can be numbered as follows. From the zeroth segment $(s=0)$ pinned at $(r=1)$ to segments in the first $j=1$ generation, $s=1, \ldots, S+1$, for the $j=2$ generation there are numbers $s=S+2, \ldots, 2(S+1)$, etc.; until at the end of generation $j=g$ there are ranking numbers $s=(g-$ 1) $(S+1)+1, \ldots, g(S+1)$. The free ends thus have the ranking number $s=g(S+1)$.

Typically, the volume fraction distribution is computed starting with the backward propagators at one of the free ends of the dendrimer. As this end is not restricted in space, the end point distribution function is integrated over all possible starting positions $G(r, s \mid g(S+1))=\sum_{r_{g(S+1)}} G\left(r, s \mid r_{g(S+1)}, g(S+1)\right)$. The backward propagator is initiated by $G(r, g(S+1) \mid g(S+1))=$ $G(r)$ for all $r$, and then propagate toward the root segment. For segments of generation $j=g$

$G_{g}(r, s \mid g(S+1))=G(r)\left\langle G_{g}(r, s+1 \mid g(S+1))\right\rangle$

that is, for $s=g(S+1)-1, \ldots,(g-1)(S+1)+1$. Here, a sub-index was added to $G$ to point to the generation the end point distribution "belonged" to. Going inward, $f-1$ chains will merge at the branch point and the following equation is re-initiated:

$$
\begin{aligned}
& G_{g-1}(r,(g-1)(S+1) \mid g(S+1))=G(r) \\
& \quad\left\langle G_{g}(r,(g-1)(S+1)+1 \mid g(S+1))\right\rangle^{f-1}
\end{aligned}
$$

With this, one proceeds to generation $j=g-1$

$G_{g-1}(r, s \mid g(S+1))=G(r)\left\langle G_{g-1}(r, s+1 \mid g(S+1))\right\rangle$

for $s=(g-1)(S+1)-1, \ldots,(g-2)(S+1)+1$. Then again, as other (equivalent) chain fragments join the walk, reinitiation is needed and one can proceed with $j=g-2$, etcetera, until generation $j=1$ is finished and finally $s=0$ is reached. For this segment, one needs to use $G_{0}(r, 0 \mid g(S+1))=G(r)$ $\left\langle G_{1}(r, 1 \mid g(S+1))\right\rangle^{f}$. As the root segment is pinned in the origin $(r=1), G_{0}(r, 0 \mid g(S+1))$ is set to zero for $r \neq 1$. At 
this point the partition function $q_{D}=G_{0}(1,0 \mid g(S+1)) L(1)$ can be evaluated. For the solvent, the partition function is $q_{O}=\sum_{r} L(r) G(r)$.

When $r=1$, the forward propagator starts with: $G_{1}(r, 0 \mid 1,0)=G(r)\left\langle G_{1}(r, 1 \mid g(S+1))\right\rangle^{f-1}$ otherwise it is initialized with zero. The next step is to propagate through the first generation $j=1$ :

$G_{1}(r, s \mid 1,0)=G(r)\left\langle G_{1}(r, s-1 \mid 1,0)\right\rangle$

for $s=1, \ldots, S+1$. Before continuing with $j=2$, the $f-2$ branches of $G_{2}(r, S+2 \mid g(S+1))$ have to be "added-on." Hence, the end point distributions are re-initiated:

$\left.\left.G_{2}(r, S+1 \mid 1,0)=G_{1}(r, S+1 \mid 1,0)\left\langle G_{2}(r, S+2)\right| g(S+1)\right)\right\rangle^{f 2}$

and continuing toward the next branch point:

$G_{2}(r, s \mid 1,0)=G(r)\left\langle G_{2}(r, s-1 \mid 1,0)\right\rangle$

for $s=S+2, \ldots, 2(S+1)$. This procedure is repeated for each generation until for the last generation the free end is reached with $G_{g}(r, g(S+1) \mid 1,0)$.

Combining the forward and backward end point distribution functions gives the volume fraction profiles:

$\varphi_{j}(r, s)=\frac{f(f-1)^{j-1}}{q_{D}} \frac{G_{j}(r, s \mid 1,0) G_{j}(r, s \mid g(S+1))}{G(r)}$

The distribution of segment $s=0$ is zero everywhere except in layer $r=1$ where it has the value $\varphi(1,0)=1 / L(1)$. The overall volume fraction distribution for the dendrimer in the cell model is found by summing over all segment ranks $s$ from the root to the ends. The index $j$ can then be dropped. The segments that belong to a part of the molecule can also be collected, for example, for a particular sub-generation $j$ or a branch point (the last segment of a sub-generation). In the literature ${ }^{[4,32]}$ more details about dendrimers in the SF-SCF cell model can be found.

\subsection{Data Analysis}

The overall density distribution relative to both the root segment and the center of mass, and the distribution of the branch and end points relative to the root segment were determined. For the FJC model, the distribution of the branch- and end points could also be evaluated relative to the center of mass. The classical way to compare different models is to compute the radius of $R_{g}$ which is a measure for the size of the dendrimers. This quantity is defined as

$R_{g}=\left\langle\left(\frac{1}{N} \sum_{h=1}^{N}\left(\mathbf{r}_{\mathbf{h}}-<\mathbf{r}>\right)^{2}\right)^{\frac{1}{2}}\right\rangle$

Here $N$ is the total number of monomers/particles in the system, $<\mathbf{r}>$ the average position/center of mass and $r_{h}$ is the coordinate of the particle $h$.
In the cell model, the dendrimers are by definition radially symmetric. In practice it is known that deviations from the spherical shape are important. Following the definition of Rudnick et al. ${ }^{[3]}$ the asphericity for the MC-FJC and MC-SCF methods was computed. This measure indicates how different the size of the object is in the three perpendicular directions. One of the axis points in the direction in which the object is the largest and one in the direction in which the object is the smallest. The third axis is perpendicular to these two. The first step to determine the asphericity is to calculate the gyration tensor $T$.

$T_{i j}=\frac{1}{N} \sum_{h=1}^{N}\left(\mathbf{r}_{\mathrm{hi}}-<\mathbf{r}_{\mathrm{i}}>\right)\left(\mathbf{r}_{\mathrm{hj}}-<\mathbf{r}_{\mathrm{j}}>\right)$

Here $i$ and $j$ are the spatial directions and $r_{h i}$ thus indicates the position of particle $h$ on the $i$ axis. Subsequently, the eigenvalues $\lambda$ of the matrix $T$ are determined. The magnitude of the eigenvalues is a measure for how extended the object is in each extremal direction. Next, the asphericity $A$ is calculated as:

$A=\frac{\sum_{i=1}^{d-1} \sum_{j>i}^{d}\left(\lambda_{i}-\lambda_{j}\right)^{2}}{(d-1)\left(\sum_{i=1}^{d} \lambda_{i}\right)^{2}}$

Here $d$ is the number of spatial dimensions.

\section{Results and Discussion}

In Figure 2, we show results for a dendrimer with $f=5, g=4$, $S=20$. Panel a is a snapshot of the MC-FJC model. For the MC-FJC model, the branch points including the root segment, which is just visible in the center, are colored red. Panel b is a snapshot of the MC-SCF model. The branch and end points are visible as orange crosses and white dots with an orange center, respectively. They are easily found as the segment density around these points is high. For illustrative purposes, we present the cell model (panel c), in an unusual way, as a density plot. The density drops from high density in the core to low density in the corona. There are many similarities between the first two images. Both dendrimers are roughly spherical, but show significant density fluctuations in azimuthal direction. Such fluctuations are not present in cell model (panel c) due to the mean field approximation. The spacers are distributed over all the possible conformations and are visible as the bluish aura around the branch and end points. Only panel a is a true single conformation snapshot because all segments have a specific position.

In Figure 3a, the radius of gyration $R_{g}$ of linear chains is shown for different models. The linear chain is a special dendrimer, namely with $f=2$ and it is interesting to consider this case first. The result for a linear chain is the well known $R_{g} \propto N^{\alpha}$, with $\alpha$ going toward 0.588 in the high chain length limit. ${ }^{[34,35]}$ The MC-FJC approaches this value from above, as in the short chain limit of just two beads it behaves as a rigid rod. The cell model on the other hand approaches 

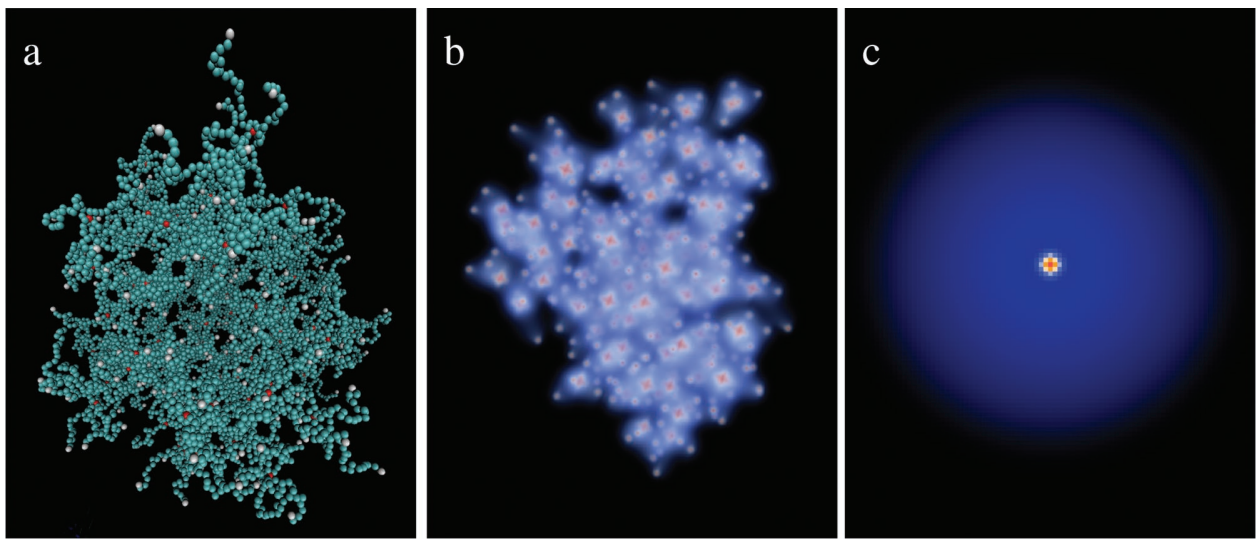

Figure 2. Snapshots of a dendrimer with $f=5, g=4, S=20$ : a) the MC-FJC model. The branch points are colored red and the end points white, b) the MC-SCF model, and c) the cell model. For (b) and (c), the segment density decreases going from orange, through white to blue and black (the scale differs between the images).

from below. Because it allows backfolding, the cell model can behave as a Gaussian coil for small chain lengths and it therefore approaches from 0.5 . For the MC-SCF model $\alpha$ is about 0.59 and numerical inaccuracies do not allow us to pinpoint whether it approaches from above or below. No significant difference between middle block lengths of $S=20$ and $S=50$ was observed, although the large confidence intervals could still hide small differences between the two.

It is interesting to point out that the value for the radius of gyration $R_{\mathrm{g}}$ for the MC-SCF model is the smallest of the three models and that MC-FJC gives the largest value. Due to the fact that for the spacers backfolding is allowed, in both the cell
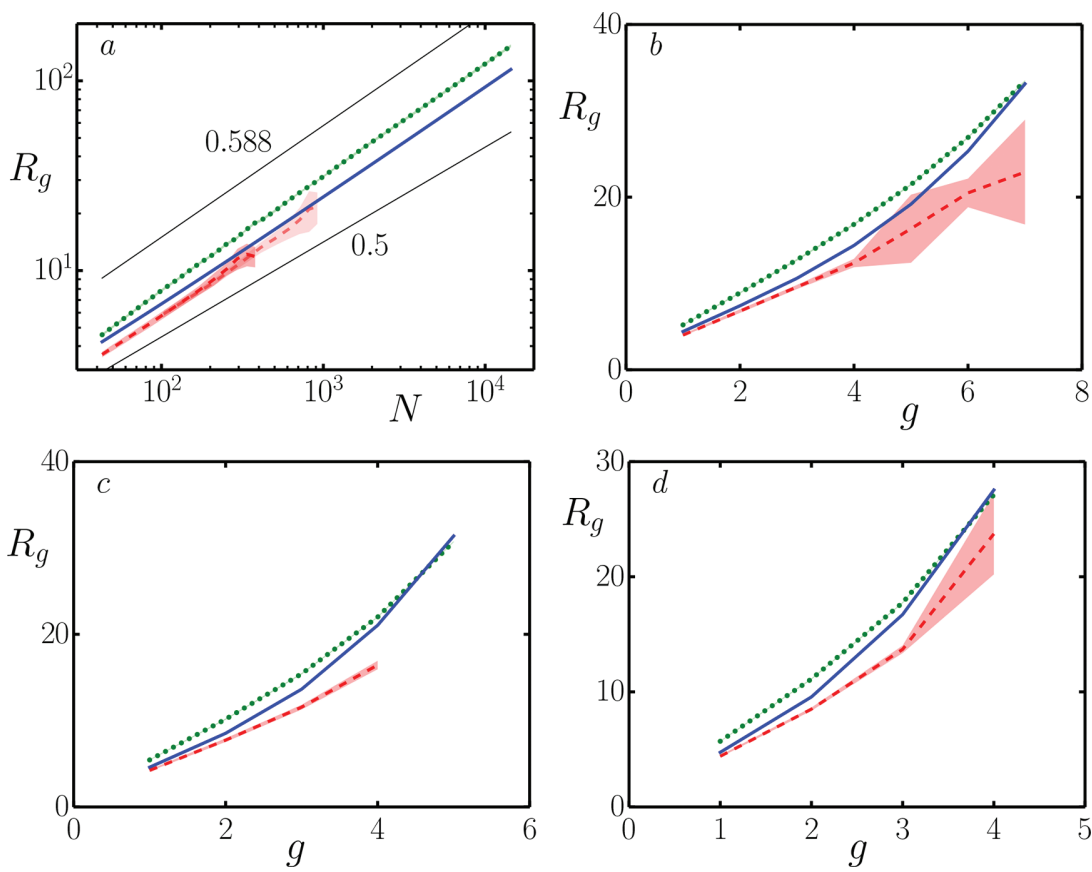

Figure 3. Radius of gyration in units / as a function of: a) the overall degree of polymerization $N$ for linear chains $(f=2)$ and b) $f=3$, c) $f=4$, d) $f=5$ as a function of the generation number

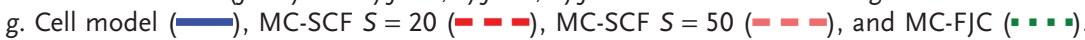
The lighter areas indicate the $99 \%$ confidence interval. model and the MC-SCF model, they give a smaller $R_{g}$ than the MC-FJC model. The cell model also overestimates the long range steric hindrance and therefore the chain is bigger than in the MC-SCF model.

In Figure $3 \mathrm{~b}-\mathrm{d}$, the radius of gyration $R_{\mathrm{g}}$ is shown for dendrimers with $f=3, f=4$, and $f=5$, respectively, as a function of the generation number $g$. Let us focus first on the difference between the least accurate (cell model) and the most accurate (MC-FJC) model. For low generation numbers $g$ the cell model (solid line) underestimates the size significantly. As the number of generations increases the cell model catches up with the MC-FJC model and for $f=4, g=5$ and $f=5, g=4$ the radius of gyration exceeds that of the MC-FJC model. As we mentioned before, this more rapid growth of the radius of gyration is likely caused by the overestimation of the steric repulsion between the branches of the dendrimer. In a real polymer, the segments are next to each other and their excluded volumes will overlap. In the cell model, the segments are distributed homogeneously within the lattice layers and there is little overlap of the excluded volumes, hence the stronger stretching. For a small number of generations, the underestimation of the short-range excluded volume wins and causes the too small size relative to the MC-FJC model.

At first sight, it may seem that the hybrid MC-SCF model is even worse, as it consistently gives a too small size for the dendrimer. The shapes of the curves are, however, very similar to that of the MC-FJC model. If the radius of gyration of the $\mathrm{MC}-\mathrm{SCF}$ model is multiplied with about 1.31 for $S=20$ and 1.37 for $S=50$ the curves overlap. The scaling behavior of the MC-SCF model should therefore be very similar to the FJC model although the relatively large confidence interval of the MC-SCF model leaves some room for deviations. 
In the literature, ${ }^{[16,17]}$ there exists a prediction for the powerlaw dependence of the size of dendrimers, $R_{\mathrm{g}} \propto(\mathrm{Sg})^{\frac{2}{5}} N^{\frac{1}{5}}$. Therefore the data on the radius of gyration have been replotted in Figure 4, as a function of $\mathrm{g}^{2} n_{\text {spacer }}$ and rescaled with a factor $\frac{1}{(S)^{0.588}}$. In Figure 4a, the results for the MC-FJC model are displayed. The curves of the dendrimers for $f=3, f=4$, and $f=5$ all lie on top of each other. The radius of gyration is thus proportional to a factor $\left(\mathrm{g}^{2} n_{\text {spacer }}\right)^{\alpha}$. A value of 0.212 for $\alpha$ gave a slightly better fit than the theoretical prediction of $\frac{1}{5}$. For the linear chains, $\alpha=0.198$ gives the best fit. As the radius of gyration of real polymer coils scales with the number of segments to the power 0.588 and the number of segments is proportional to the number of generations. This value is in between the realistic value of 0.196 and the value of $\frac{1}{5}$ from the theoretical predictions. In Figure 4b, we have plotted the radius of gyration for the MC-SCF model. The curves for the dendrimers again seem to lie on top of each other although they do not match quite as well as for the MC-FJC model. Due to the relatively large uncertainty margins, we cannot conclude that the scaling differs significantly from the MC-FJC model. Finally, in Figure 4c, we have plotted the data for the cell model. The curve for $f=3$ no longer matches up with those of $f=4$ and $f=5$. The cell model also predicts a much higher value of 0.244 for $\alpha$. It is thus clear that the cell model does not show accurate scaling for these dendrimers.
Let us next focus on the average distance of the end segments from the central segment. Even though it is expected that this quantity has similar information as the radius of gyration, we present a selection for these distributions in Figure 5. As in the previous Figure, we focus on panel a on the $N$-dependence for $f=2$ (linear chains) and on panel b on the dependence on the generation number $g$, for $f=3$ and note that for $f=4,5$ similar trends were obtained.

For the end point distance, one would again expect a powerlaw dependence. To a reasonable approximation, this indeed happens, albeit that in all cases we see a somewhat larger deviation from the expected limiting power law value of 0.588 than for the radius of gyration (cf. Figure 3a), especially for the MC-SCF model for which the exponent is about 0.65. These deviations are again attributed to the finite chain lengths. Apparently we should view the MC-SCF chain as a bead spring model, with each branch and end point as a bead. These beads would interact through soft potentials generated by the spacers. As the number of beads in MC-SCF is very small we see large deviations from the limiting values. We also see such large deviations for the end point distances of the MC-FJC model when very short spacers are used. Just as for the MC-SCF model, these differences are larger for the end point distances than for the radius of gyration.

In Figure 5a, we show results for both $S=20$ and $S=50$ for the MC-SCF model. The larger the value of $S$, the fewer MCbeads $(N / S)$ we have in the simulation. We noticed that the average position of the end points differs between $S=20$ and $S=50$. The curve for $S=50$ starts below the curve of $S=20$
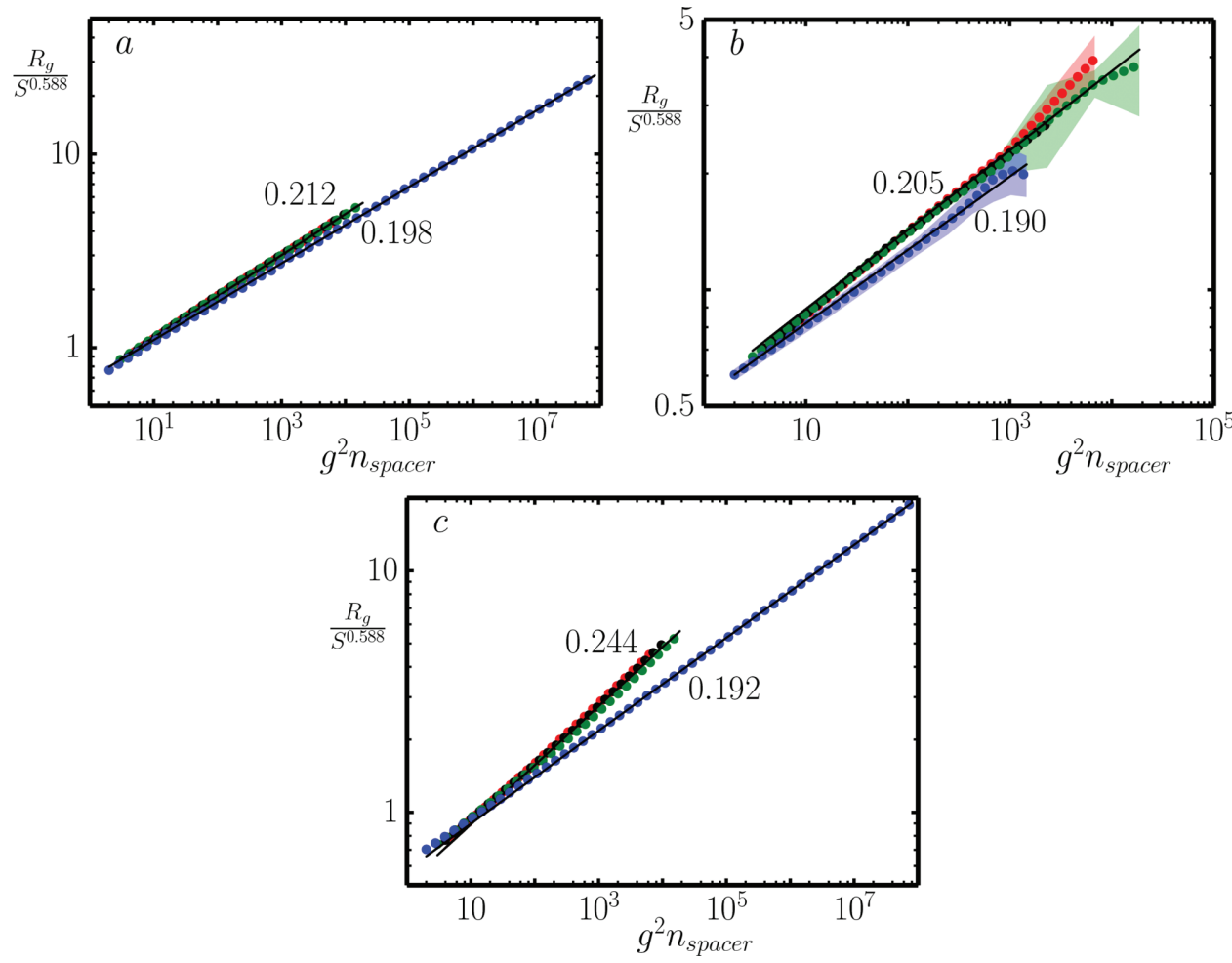

Figure 4. Radius of gyration a) MC-FJC b) MC-SCF, c) cell model, $f=2(\bullet \bullet \bullet), f=3(\bullet \bullet \bullet), f=4(\bullet \bullet \bullet), f=5(\bullet \bullet \bullet), S=20+1$ (the segment that forms the branchpoint also needs to be counted, thus +1$)$. The black lines indicate the best fits for $\left(g^{2} n_{\text {spacer }}\right)^{\alpha}$. The black numbers indicate the fitted value of $\alpha$. The lighter areas indicate the $99 \%$ confidence interval. 

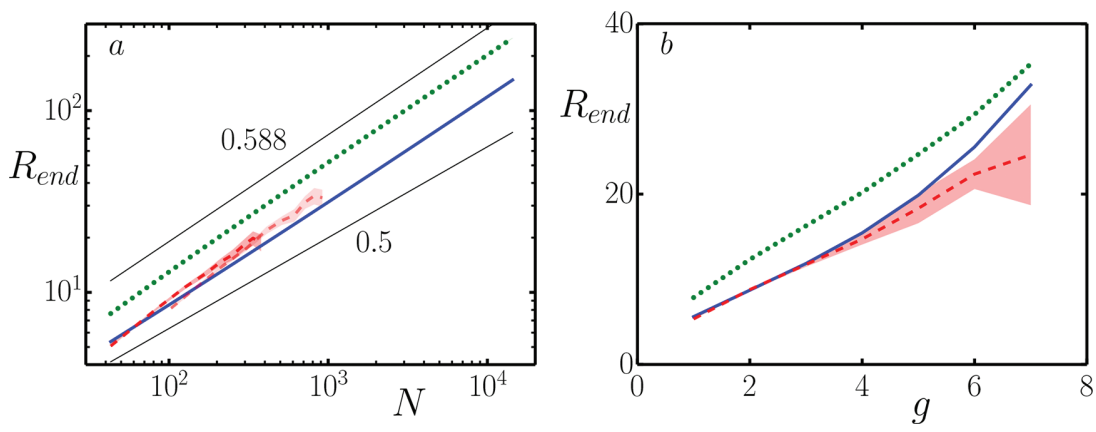

Figure 5. The average end segment distance relative to the central segment $R_{\text {end }}$ in units / for: a) linear chains $(f=2)$ as a function of $N$; b) for $f=3$ as a function of the generation number g. Cell model ( $(-)$, MC-SCF $S=20(---)$, MC-SCF $S=50(---)$, and MC-FJC (" " " "). The lighter areas indicate the $99 \%$ confidence interval.

and this difference diminishes with increasing $N$. This implies that the corresponding power-law coefficient is larger for $S=$ 50 , which is expected if the MC-SCF chains are viewed as a bead spring model. Recalling that the radius of gyration was the same for these two cases, it implies that the radial density distribution should be different.

In Figure 6, we present the radial density profile for two linear chains with the same number of segments $N=63$ modeled by the MC-SCF hybrid. The two results differ with respect to the number of MC-beads that were implemented. Close inspection of Figure 6 shows that the curves for $S=20, g=3$ and $S=62, g=1$ cross each other twice. At very short and large distances $r$ from the central segment the volume fraction $\varphi$ for $S=20, g=3$ is higher than for $S=62, g=1$. At intermediate distances the opposite is true. These two trends have the effect that $R_{g}$ is approximately the same for both, but as the end-segments are further away from the center, the largest end point position is found for $S=20, g=3$.

A possible reason for this difference between $S=20$ and $S=62$ is that for $S=62$ it costs less entropy to go a few steps in the "wrong" direction. The blobs of density around the branch points are therefore larger for $S=62$ than for $S=20$. In the center of the blob, the behavior will be similar to the cell model. That is, the density of the polymer chain is still smeared around the branch point, because there are still a fair number of conformations that move away from the next branch point in the first few steps on the lattice. Similar to the cell model, the

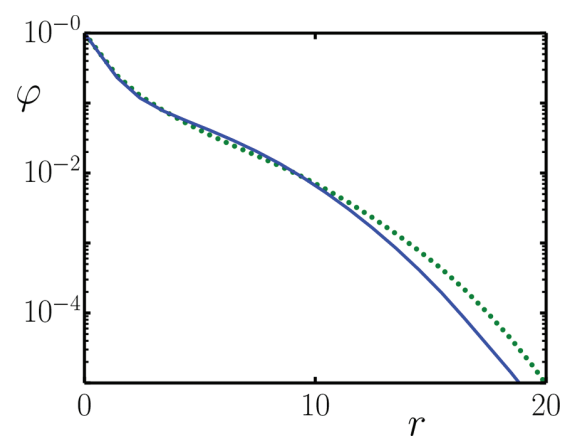

Figure 6. The average radial density profile relative to the central segment as found by the MC-SCF model for $f=2$. The two cases have the same value of $N: S=62, g=1$ ( $), S=20, g=3(" \cdot ")$ ). ties are high. repulsion between different branches around the branch point is therefore overestimated. The area for which this effect takes places is larger for $S=62$ and therefore the spacers are stretched more near the central branch point which results in a faster decrease in density near the central segment. Near the end point there is only one chain and this effect is much weaker. The longer spacer 62 can spread itself over a larger area and therefore the repulsion between segments next to each other in the spacer is less. The result is that the chain is less stretched near the ends and the average end point distance is therefore smaller.

The average end point distance for $f=3$ is shown in Figure 5b. In general the trend for the end point distance is the same as for the radius of gyration. Again we see that the full excluded volume MC-FJC result gives the largest dendrimer size. For low values of $g$ the cell model and the MC-SCF do not differ much. For large g, the cell model goes toward the MC-FJC result and it is expected that it will overtake this for sufficiently large $g$. The size of the dendrimer in the MC-SCF model stays more systematically below that of the MC-FJC model, suggesting once again that there is just a different prefactor. Close inspection reveals that a similar shift as reported above for the $R_{g}$ results does not give the same quality of overlap of curves in this case.

In Figure 7, the radial density profiles, relative to the central segment, are shown for a functionality $f=2,3,4,5$ with $S=$ 50 for $f=2$ and $S=20$ for $f>2$, and respective generations $g=9,7,4,4$. For the linear polymers (panel a) the density drops monotonically and the inner region can be approximated by a power law $\varphi(r) \propto r^{\beta}$. For all three models, $\beta \approx-\frac{4}{3}$, with a slightly larger value for the MC-FJC model and a slightly smaller value for the cell model. This is consistent with the work of de Gennes, ${ }^{[36]}$ who also predicted a value of $-\frac{4}{3}$. For the MC-SCF model, there seems to be a slight kink in the early part of the curve which may be related to a change in behavior going from length scales less than the inter node distance to length scales larger than the inter node distance. We should be careful not to over interpret these numbers because we definitely need better statistics to find accurate values for the density in and around the center. For MC-FJC an irregular distribution is found in the very center. Near the center the branches are strongly stretched, the second segment from the center therefore is mostly in the positions farthest from the center. This results in a peak at $r=2$. These effects are frequently seen in simulations when excluded volume effects are truly accounted for and local densi-

Inspection of Figure $7 \mathrm{~b}-\mathrm{d}$, shows that for both the MC-SCF and the MC-FJC model, there exists a clear dip in the density profile of the dendrimers. There is only a marginal dip of approximately $3 \%$ for the cell model. This is in line with the results of Klein Wolterink et al., ${ }^{[32]}$ who did not observe a dip for neutral dendrimers in a cell model. According to our simulations and in line with other dendrimer simulations, ${ }^{[19,37,38]}$ a 

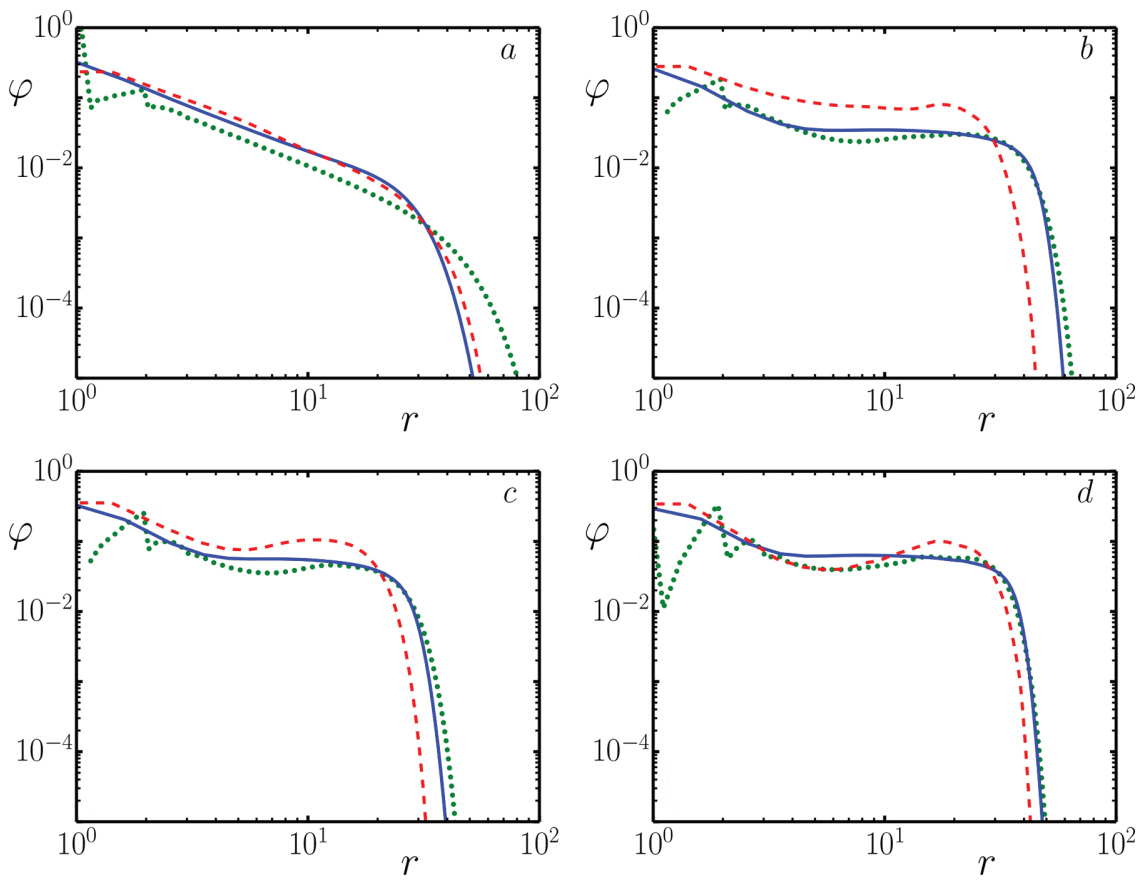

Figure 7. Radial density profiles relative to the central segment for a) $f=2, g=9, S=50$; b) $f=3$, $g=7, S=20$; c) $f=4, g=4, S=20$; d) $f=5, g=4, S=20$. Cell model ( $\square$ ), MC-SCF ( $\square=$ ), and MC-FJC (י: י י).

true dip in the radial density only occurs for systems with strong steric hindrance between the spacers, that is, for $f=3, g>5, f=$ $4, g>3$, and $f=5, g>2$. The relative depth of this dip increases as the number of spacers per branch point $f$ or the number of generations $g$ is increased. The largest dip depth, for the MC-FJC model, is for $f=5$ and $g=4$ where the density is only $65 \%$ of the maximum further out. As mentioned, the results for the MC-SCF model are also consistent with a non-monotonic density profile, but numerical uncertainties in the local density prevent us from quoting a numerical value for the depth. Computer simulations by Chen et al. ${ }^{[18]}$ who used a similar MC-FJC model with similar trial moves did not report a dip in the radial density profiles. Because these authors used slightly smaller beads than our FJC model and their results were noisier than our curves, they may have missed the dip in density.

Let us next consider a pair of key structural features inside the dendrimer. The first of these is the end point distribution. Naïvely one could expect that the end points are solely distributed at the outside of the dendrimer, but this view has been rejected and it is commonly understood that the ends are distributed throughout the dendrimers. Nevertheless it is interesting to see which distribution our models predict for the dendrimer.
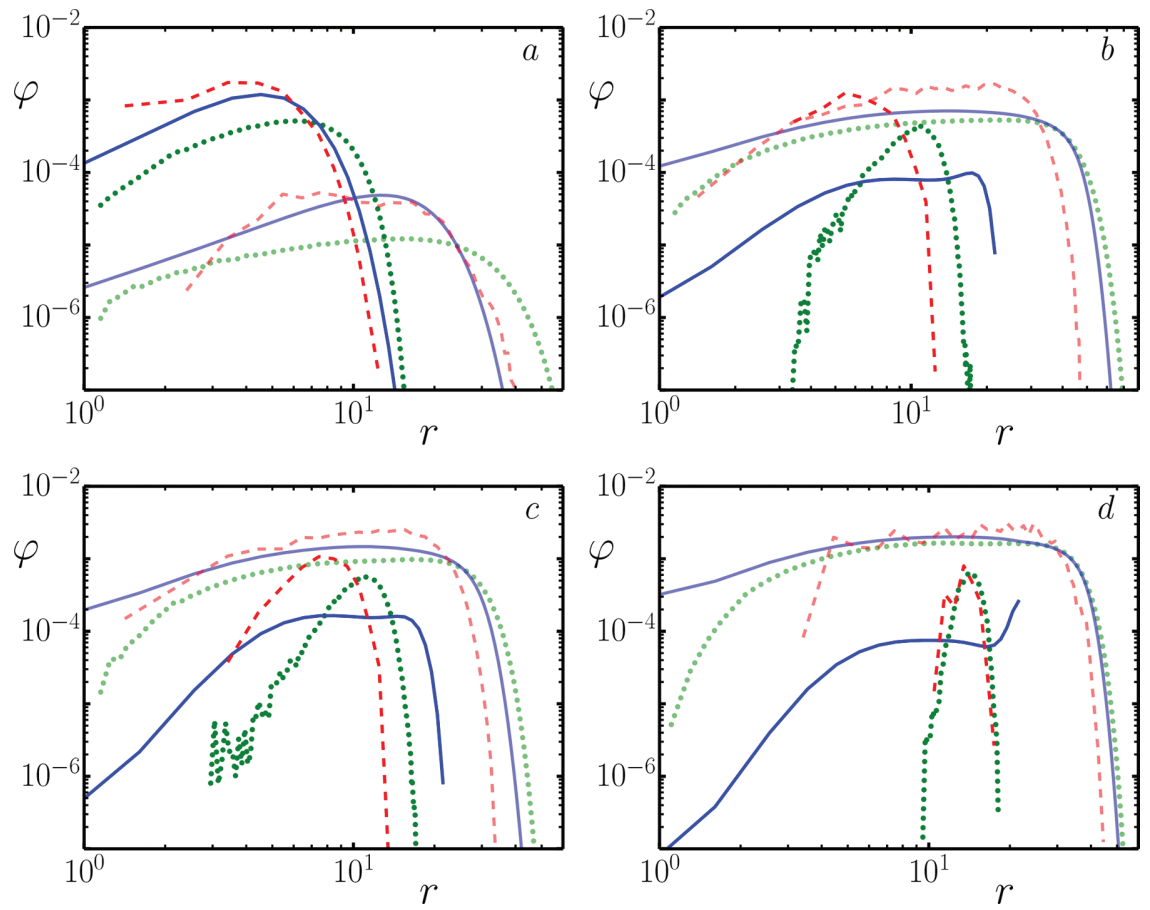

Figure 8. The volume fractions of the first branch point (dark) and end points (lighter), relative to the position of the central segment, for a) $f=2, g=9, S=20$; b) $f=3, g=7, S=20$; c) $f=4, g=4$,

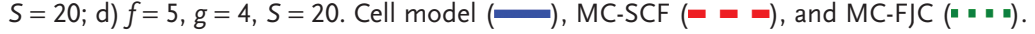


placed exactly in the origin. In the cell model the central segment is, however, placed in the first layer around the origin. The central segment is therefore half a lattice site away from the origin and the density in the center is thus lower. Another explanation is that the last generation of branch points is on average further from the center for the Monte Carlo models than for the cell model. The chains thus have to stretch more to reach the center for the Monte Carlo models.

The results for the distribution of the first off-center branch point are much more interesting. Inspection of the darkly colored curves in Figure 8 shows that the profiles resemble each other reasonably well for the linear chain, but deviate progressively more as the molecular crowding increases. There is just one trend that is the same for al models, namely, the outward shift of the branch points with increasing crowding. With the cell model (solid blue lines), we notice an initial increase in the width of the profiles and for stronger crowding (large $f$ and g-values) the distribution becomes clearly bimodal. A similar bimodal branch distribution has been observed previously by both Polotsky et al. ${ }^{[5]}$ with a SF-SCF model of brushes of dendrimer arms with $g=2,3,4, S=100$, and $f=2,3,4,5,8$ and Rud et al..$^{[4]}$ who did a study on dendrimer arms attached to surfaces of various curvature, again using the cell model. The results of Klein Wolterink et al. ${ }^{[32]}$ also show a peak near maximum extension for the distribution of the first branch points. Due to the way they plotted the data, it is, however, not clear whether a second peak is also present. Merlitz et al. ${ }^{[39]}$ used a Langevin dynamics simulation to study brushes made of dendrimer arms of a dendrimer with $S=50, f=4$ and $g=2$. They observed two peaks in the density distribution of the end points and from this they also concluded there were two populations.

The common interpretation of this bimodal distribution for brushes is that there are two conformation populations for the dendrimers. The first population has a weakly stretched first spacer, and its segments fill up the density near the surface. The second population has a fully stretched first spacer and these conformations provide the segment density at the outside of the brush. In our case we see similar effects inside one dendrimer. The interpretation therefore is that the different arms connected to the central branch point have different conformations. The arms with weakly stretched first spacer are mostly in the interior of the dendrimer, the arms with a fully stretched first spacer make up most of the outside of the dendrimer.

This cell model result should be compared to predictions of the MC-FJC model. With increasing crowding (higher $f$ and $g$ values, i.e., going in Figure 8 from panels $b$ to $d$ ) the profile of the first branch point sharpens while it shifts outward. Unlike the cell model, the position of the maximum does not reach the fully extended value. At very large crowding (panel d) the maximum is positioned in between the two maxima found by the cell model, not far from the local minimum. In stark contrast to the cell model, there is thus no tendency to go to a bimodal distribution with increased crowding.

The MC-SCF hybrid follows the result of the MC-FJC model. Also with this method it is found that the distribution of the first generation of branch points sharpens and moves to higher $r$ values upon an increase of $f$ and $g$. Inspection of panel $d$ shows that MC-SCF and the full MC model give virtually the same profile for the branch points. While at intermediate crowding (panels b and c) the distribution of the branch points for the MC-SCF has a similar shape as the MC-FJC model, although the spacers are less stretched. Within the MC-SCF model there is also no trend toward bimodality of the branch point distribution.

Interestingly, at intermediate crowding, both MC-models gradually develop a so-called dead zone, that is, a region near the center where no branch points are found. Such a dead zone is not present in the cell model. In MC-FJC and MC-SCF, the dead zone widens with increasing crowding. We can only conclude that in neither there are indications for two populations of dendritic arms in a given dendrimer.

There are several effects that together may have caused the deviating behavior for the SCF cell model. As we have stated before the steric repulsion with other branches is overestimated in the cell model. The force to stretch the spacers will therefore be a bit stronger in the cell model. This may have triggered the 'transition' toward two populations. As the shortrange excluded volume is underestimated by SCF, the fragment that is "retracted" can do so more easily than in reality and this might have stabilized the two-population state.

Yet another reason is that in the cell model it is possible to retract, for example, 0.5 dendrimer arms, while in the more realistic MC-FJC and MC-SCF models only whole arms can be retracted. Of course, we cannot exclude the possibility that for higher functionalities and/or a larger number of generations, one of the arms folds inward completely and effectively fills the dip in density as we have seen in Figure 7. In that case the MC-SCF and MC-FJC models may thus also develop two populations.

Interestingly, for weakly dissociating polyelectrolyte stars, Uhlik et al. ${ }^{[40]}$ have also observed a wide and bimodal distribution of end points for the SF-SCF model. Monte Carlo simulations, however, showed a much narrower unimodal distribution. ${ }^{[40]}$ For a poor solvent, they, however, did find a bimodal distribution. ${ }^{[41]}$ They ascribed this difference to the inaccurate way in which the SCF method accounts for intra-molecular excluded volume, but did not go into detail. Arguably, the conservative advise therefore is to approach bimodal distributions found by SCF theory with caution, especially when there is not a clear mechanism that can support bimodality (e.g., in "gas""liquid" equilibria in microphase segregation).

So far, we have been referring several times to the (a)sphericity of the dendrimers. With increasing $f$ and $g$, one would expect that the asphericity, which is a measure for the deviation from a spherical shape, would decrease. We therefore end our analysis by considering this property for linear chains and dendrimers. In Figure 9, we present for $f=2,3,4$, and 5 the asphericity $A$ as a function of the generation number $g$, for spacer length $S=20$. Again, for $f=2$ (linear chains) this means that there are $2 g+1$ MC-beads in the MC-SCF model and the degree of polymerization is $N=2 g(S+1)+1$. In the cell model, the spherical symmetry is imposed and therefore the cell model always has an asphericity of zero. As a result, we can only discuss the performance of the MC-SCF hybrid (dashed lines) in relation to the FJC result (dotted lines).

Inspection of Figure 9 shows that the MC-SCF hybrid performs very well with respect to fluctuations in shape. For the dendrimers $(f>2)$ in Figure $9 b-d$, two generations are enough 

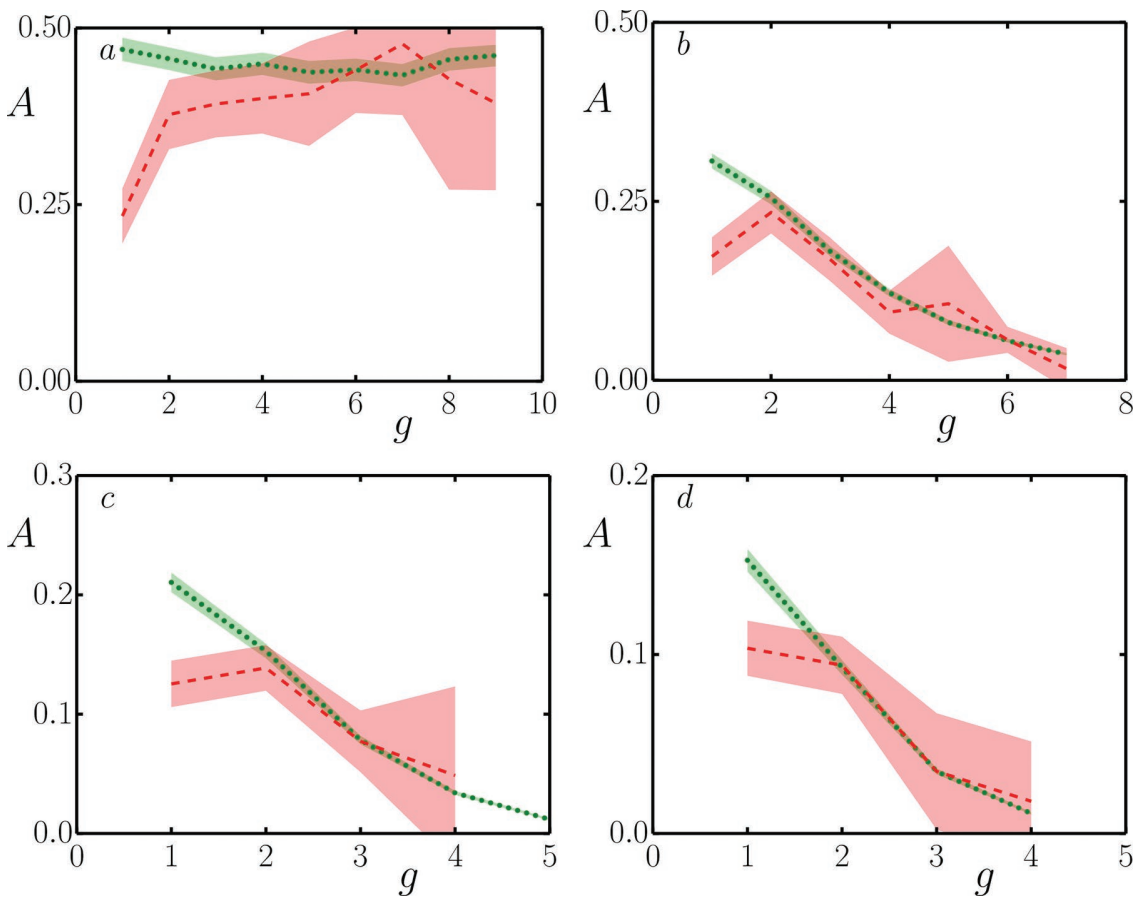

Figure 9. The asphericity for a) $f=2, S=20$; b) $f=3, S=20$; c) $f=4, S=20$; d) $f=5, S=20$. MC-SCF $(--)$ and MC-FJC ( - " $)$. The lighter areas indicate the $99 \%$ confidence interval.

to let the difference in asphericity with the MC-FJC model become less than the uncertainty margin. For linear polymers, a few more branch points are needed before the asphericities are the same, within the uncertainty margins, as those of the FJC model. Although the confidence interval is still quite large, the MC-SCF model thus seems to capture the asphericity of the dendrimers well. In both models we see that for linear chains the asphericity goes to roughly a value of 0.4 and as expected the values for $A$ tend to be a strongly decreasing function of the number of generations in the dendrimer $g$. The absolute values of $A$ decrease with increasing $f$.

\section{Conclusion and Outlook}

With the SF-SCF theory properties of complex polymeric systems can be obtained in seconds of CPU time. The SF-SCF theory, however, does not treat the correlations between the polymer segments correctly. This may in turn have consequences for interactions which strongly depend on such correlations, such as excluded volume interactions. To asses how big the errors due to neglecting part of the correlations are, we used dendrimers as a model system, because excluded volume interactions played an important role in them. In an attempt to bring back some of the correlations in the SF-SCF theory, we created the MC-SCF model. In this model, the positions of some of the segments are specified while the remaining segments are treated with the SF-SCF theory. Subsequently, the segments were moved with a Monte Carlo algorithm. As a reference system we used a freely jointed chain model (MC-FJC) with hard beads. Both the cell model and the MC-SCF model show quantitative differences with the MC-FJC model. More importantly though the cell model also shows qualitative differences such as a different scaling for the radius of gyration of the dendrimers. We also found that the cell model predicts a bimodal distribution for the first branch point from the center for large $f$ and $g$. This suggests that there are two populations of conformations inside the dendrimer. These were, however, not found by the other two fundamentally more correct approaches. We attribute the differences between the results of the MC-SCF and the MC-FJC model and those of the cell model, to an overestimation of the long range- and underestimation the short-range excluded volume interactions in the cell model. Together with the property that in SCF the bimodality can erroneously be created by "fractional" branch-point redistributions, this can explain why a two sub-population status is found for the cell model when both $f$ and $g$ are large. This flaw of SCF in the high crowding limit came as a surprise, because in the high crowding limit (large $f$ and $g$ values) one usually finds that the flaws of the mean field approximation are relatively small. In general one should thus be careful when interpreting results from the cell model. The MC-SCF model is fundamentally better at describing dendrimers than the cell model, as it gives the correct qualitative behavior. It is, however, computationally far more costly than the cell model. In an attempt to keep the CPU time within bounds we introduce the concept of "sub-boxes." These sub-boxes are significantly smaller than the overall volume and this allows to compute the segment densities of fragments of the molecule on a "local" level. Even with this implementation trick, the MC-SCF method was computationally expensive. In the current implementation, the MC-FJC model was considerably faster than the MC-SCF model. For the MC-FJC model, we use wheel moves while for the MC-SCF moves small displacements are used. The wheel moves, however, displace the branch points over considerably larger distances and therefore equilibrate the structure more quickly. In hindsight, we realize that these moves should also have been implemented for the MC-SCF model. A quick try for $F=3, g=7$, and $S=20$ showed that by doing wheel moves, $50 \%$ of the time, the number of sampled configurations could be increased by a factor 13 .

We did not use an explicit solvent in the current dendrimer models. If, however, an explicit solvent would have been present the acceptance rate of the large wheel moves in the MC-FJC model would be practically zero, because the beads would overlap with solvent molecules. We therefore expect that the difference in CPU time between MC-SCF and the MC-FJC method will be much less for denser systems, such as mixtures of polymeric molecules. For the MC-SCF model, there are also no extra cost if instead of one polymer there would be two polymers between the branch points. For dendrimers this would be very unusual but in polymeric gels there will be spacers that 
connect to the same branch points. Together with possible extra optimization steps in computing the volume fractions per spacer (better implementation of sub-boxes) and smarter Monte Carlo moves we expect that for dense polymeric systems the MC-SCF hybrid can outperform simulation methods that take all excluded volume interactions explicitly into account.

\section{Supporting Information}

Supporting Information is available from the Wiley Online Library or from the author.

\section{Acknowledgements}

This research was funded by NWO, The Netherlands Organization For Scientific Research.

\section{Conflict of Interest}

The authors declare no conflict of interest.

\section{Keywords}

dendrimers, excluded volume, freely jointed chain, Monte Carlo simulation, Scheutjens-Fleer, self consistent field theory (SCF)

Received: November 23, 2018 Revised: March 20, 2019

Published online:

[1] A. J. de Graaf, I. I. A. P. dos Santos, E. H. E. Pieters, D. T. S. Rijkers, C. F. van Nostrum, T. Vermonden, R. J. Kok, W. E. Hennink, E. Mastrobattista, J. Controlled Release 2012, 162, 582.

[2] M. Mocan, M. Kamperman, F. A. M. Leermakers, Polymers 2018, $10,78$.

[3] O. V. Borisov, A. A. Polotsky, O. V. Rud, E. B. Zhulina, F. A. M. Leermakers, T. M. Birshtein, Soft Matter 2014, 10, 2093

[4] O. V. Rud, A. A. Polotsky, T. Gillich, O. V. Borisov, F. A. M. Leermakers, M. Textor, T. M. Birshtein, Macromolecules 2013, 46, 4651.

[5] A. A. Polotsky, T. Gillich, O. V. Borisov, F. A. M. Leermakers, M. Textor, T. M. Birshtein, Macromolecules 2010, 43, 9555.

[6] S. Pogodin, V. A. Baulin, Soft Matter 2010, 6, 2216.

[7] J. Sprakel, N. A. M. Besseling, M. A. C. Stuart, F. A. M. Leermakers, Eur. Phys. J. E 2008, 25, 163.
[8] B. R. Postmus, F. A. M. Leermakers, M. A. C. Stuart, Langmuir 2008, 24, 6712.

[9] G. H. Fredrickson, H. Orland, J. Chem. Phys. 2014, 140, 084902.

[10] P. J. Flory, Statistical Mechanics of Chain Molecules, Oxford University Press, Oxford 1988

[11] A. Ben-Shaul, I. Szleifer, W. M. Gelbart, J. Chem. Phys. 1985, 83, 3597.

[12] J. Bergsma, F. A. M. Leermakers, J. M. Kleijn, J. van der Gucht, J. Chem. Theory Comput. 2018, 14, 6532.

[13] F. A. M. Leermakers, J. Bergsma, J. van der Gucht, J. Phys. Chem. A 2012, 116, 6574

[14] M. Charlaganov, F. A. M. Leermakers, J. Chem. Phys. 2009, 131, 244115.

[15] J. van Male, Ph.D. Thesis, Laboratory of Physical Chemistry and Kolloid Science, Wageningen University, The Netherlands 2003.

[16] J. Klos, J. U. Sommer, Polymer Science Ser. C 2013, 55, 125.

[17] M. Ballauff, C. N. Likos, Angew. Chem. Int. Ed. 2004, 43, 2998.

[18] Z. Y. Chen, S.-M. Cui, Macromolecules 1996, 29, 7943.

[19] J. Klos, J.-U. Sommer, Macromolecules 2009, 42, 4878.

[20] G. J. Fleer, M. A. C. Stuart, J. M. H. M. Scheutjens, T. Cosgrove, B. Vincent, Polymers at Interfaces, Chapman \& Hall, London 1993.

[21] J. M. H. M. Scheutjens, G. J. Fleer, J. Phys. Chem. 1979, 83, 1619.

[22] J. M. H. M. Scheutjens, G. J. Fleer, J. Phys. Chem. 1980, 84, 178.

[23] O. A. Evers, J. M. H. M. Scheutjens, G. J. Fleer, PhD Thesis, Wageningen University, The Netherlands 1990.

[24] F. A. M. Leermakers, J. M. H. M. Scheutjens, J. Lyklema, Biophys. Chem. 1983, 18, 353.

[25] H. Pera, J. Kleijn, F. Leermakers, J. Chem. Phys. 2014, 140, 065102.

[26] L. Meijer, F. Leermakers, J. Lyklema, J. Chem. Phys. 1999, 110, 6560.

[27] Y. Lauw, F. Leermakers, M. C. Stuart, J. Phys. Chem. 2003, 107, 10912.

[28] J. Bergsma, F. A. M. Leermakers, J. van der Gucht, Phys. Chem. Chem. Phys. 2015, 17, 9001.

[29] I. Ionova, E. Carter, J. Compt. Chem. 1996, 17, 1836.

[30] C. M. Wijmans, E. B. Zhulina, Macromolecules 1993, $26,7214$.

[31] F. A. M. Leermakers, C. M. Wijmans, G. J. Fleer, Macromolecules $1995,28,3434$.

[32] J. K. Wolterink, J. van Male, M. Daoud, O. V. Borisov, Macromolecules 2003, 36, 6624

[33] J. Rudnick, G. Gaspari, Elements of the Random Walk, Cambridge University Press, Cambridge 2004.

[34] R. Guida, J. Zinn-Justin, J. Phys. A: Math. Gen. 1998, 31, 8103.

[35] N. Clisby, Phys. Rev. Lett. 2010, 104, 055702.

[36] P.-G. de Gennes, Scaling Concepts in Polymer Physics, Cornell University Press, Ithaca, NY 1979.

[37] J. Klos, J.-U. Sommer, Macromolecules 2013, 46, 3107.

[38] E. G. Timoshenko, Y. A. Kuznetsov, R. Connolly, J. Chem. Phys. 2002, $117,9050$.

[39] H. Merlitz, C.-X. Wu, J.-U. Sommer, Macromolecules 2011, 44, 7043.

[40] F. Uhlík, P. Košovan, Z. Limpouchová, K. Procházka, O. V. Borisov, F. A. M. Leermakers, Macromolecules 2014, 47, 4004.

[41] F. Uhlík, P. Košovan, E. B. Zhulina, O. V. Borisov, Soft Matter 2016, $12,4846$. 